\title{
Clinical Experience in the Administration of Intravitreal Injection Therapy at a Tertiary University Hospital in Jordan During the COVID-19 Lockdown
}

This article was published in the following Dove Press journal:

Clinical Ophthalmology

\author{
Omar A Saleh (D) \\ Hisham Jammal (D)' \\ Noor Alqudah (1) \\ Asem Alqudah (D)' \\ Nakhleh Abu-Yaghi $\mathbb{D}^{2}$ \\ 'Department of Ophthalmology, Jordan \\ University of Science and Technology, \\ Irbid, Jordan; ${ }^{2}$ Department of Special \\ Surgery/Ophthalmology Division, School \\ of Medicine, The University of Jordan, \\ Amman, Jordan
}

Purpose: To describe the clinical experience with the delivery of intravitreal injection therapy to patients with various indications at a tertiary university hospital during the COVID-19 lockdown in Jordan.

Methods: This is a retrospective observational study of patients who received intravitreal injections between April 12th and May 9th, 2020, a period during the national COVID-19 lockdown (March 16th to June 6th, 2020). Special medical and logistic arrangements, priority and visual risk assessment and strict infection control precautions were implemented. Demographics, diagnosis, intravitreal injection history, medical history, ophthalmic examinations and optical coherence tomography data were collected and analyzed.

Results: Intravitreal injections were successfully administered to 132 patients with diabetic retinopathy, age-related macular degeneration and retinal vein occlusion. All logistic and transmission control measures were followed by the medical staff and patients with no incidents. No new exposures or COVID-19 positive cases were traced to our location or time of therapy. No complications related to the injections were recorded. The mean period of delay due to the lockdown from the original scheduled appointment was six weeks. Mean visual acuity significantly decreased from 20/55 before the lockdown to 20/70 after the lockdown, and mean central macular thickness significantly increased from 329 to $370 \mu$.

Conclusion: The administration of intravitreal injection therapy during the COVID-19 lockdown under special safety precautions was feasible and successful. Resumption of the essential therapies and medical services during periods of pandemic restrictions while adhering to strict transmission control measures is encouraged.

Keywords: intravitreal injections, COVID-19, lockdown, diabetic macular edema, agerelated macular degeneration

\section{Introduction}

For the past few months, the world has been facing considerable challenges due to the COVID-19 (COVID-19: COrona VIrus Disease caused by the 2019-nCoV virus) pandemic. ${ }^{1}$ With the high transmissibility and with the necessity for advanced respiratory support, many medical networks across different parts of the world have been overwhelmed. The World Health Organization has declared the COVID-19 virus outbreak a pandemic on March 11th, 2020 and has devised guidelines and strategic responses to control the infection worldwide. In response, proactive efforts and
Correspondence: Omar A Saleh Department of Ophthalmology, Jordan University of Science and Technology, 3030 Ramtha Street, Irbid 22110, Jordan

Tel +962-2-754-1594

Email omarsaleh80@yahoo.com 
lockdowns designed to limit the spread of the virus were implemented in many countries. ${ }^{2,3}$ Although necessary to control the pandemic, these measures have resulted in major interruptions in healthcare, economy, social life and many public services. In Jordan, a complete lockdown was initiated on March 16th and lasted until June 6th, 2020. During the lockdown, the whole national healthcare service was curtailed to the prevention and management of COVID19-related disease and to emergency services only. In the field of ophthalmology, regular clinic visits, elective surgeries, scheduled intravitreal injections and services for nonurgent eye conditions, were all deferred.

Several recommendations and guidelines for ophthalmic practice during the COVID-19 era, including intravitreal injections (IVI), have been recently introduced by international expert committees and ophthalmology teams who had first-hand COVID-19 experience. ${ }^{4-10}$ Following such protective measures may be particularly relevant in ophthalmic practice due to the increased risk for transmission of respiratory-borne pathogens, including the COVID-19 virus, with the close proximity that is often required between patients and caregivers. ${ }^{11}$ Risk assessment and triage, postponement of non-urgent cases, hygienic practices and protective equipment as well as teleophthalmology virtualized services, have been proposed or implemented. ${ }^{6}$ However, suspending vital ophthalmic services, as in IVI, may result in significant or irreversible vision loss in some patients. ${ }^{8}$

Due to the accumulating need for IVI therapy after the lockdown, and with the uncertainty that surrounds the prevention or management of COVID-19 disease, the authors took the initiative to provide IVI therapy for priority patients during the lockdown. We aim to describe our clinical experience with IVI therapy at a tertiary university hospital during the COVID-19 lockdown. We discuss the medical and logistical arrangements we applied to invite priority patients to the hospital and administer the necessary IVI to them. We also measure progression in disease activity, if any, that may have occurred in those patients whose therapy was delayed due to the lockdown, by comparing clinical evaluations recorded before and after the lockdown.

\section{Methods}

\section{Ethical Approval, Patients and Data Collection}

We were given permission from the institutional review board at the Jordan University of Science and Technology to conduct this retrospective observational study of patients who received IVI at the university hospital during the COVID-19 lockdown, which started on March 16thand lasted until June 6th, 2020. We were given approval from the national infection control committee for providing the treatment to patients during the lockdown but with conditions, including screening for COVID-19 symptoms and exposure upon calling the patients, prioritization of the listed patients according to medical necessity, a maximum capacity of 10 patients per day, a maximum of 4 weeks during active lockdown to complete the therapy, keeping the number of medical personnel on duty to a minimum, and the strict application of predetermined infection control and social distancing protocols.

Data collected included demographics, diagnoses, dates of and type of injections, medical history, ophthalmic examination and optical coherence tomography (OCT) scans of the current and last few visits. Cataract severity was graded from +1 to +3 by the ophthalmologist. Snellen visual acuity was converted to $\log$ MAR value for statistical analysis. Based on the Freiburg Visual Acuity Test, the $\log$ MAR equivalent value for "counting fingers" was assumed to be $1.85 .{ }^{12}$ For classification of diabetic retinopathy, the 2019 American Academy of Ophthalmology "preferred practice pattern" guidelines were adopted. ${ }^{13}$

To obtain a subjective point of view from the patients' perspective on whether the delay in therapy due to the lockdown has affected their visual function or quality of life, they were asked 1 or 2 questions while in the waiting room before they received their IVI. The first question was: "How would you describe your visual function at this time compared to your last visit before the lockdown?" The patients were given three options for an answer: better, worse, no notable change. If the patient's answer was "worse", the nurse would ask the following second question: How did the delay in treatment due to the lockdown affect your quality of life?

All patients signed an intravitreal injection consent form, a written informed consent for the questionnaire and data collection for the study, and a self-declaration COVID-19 form.

\section{Risk Assessment and Prioritization of Patients}

The medical records of all the patients who were scheduled to receive IVI after the day of the lockdown and who 
missed their IVI therapy before the lockdown were reviewed to verify the diagnosis and injection and to establish a priority system based on visual loss risk assessment. Preset priority factors were searched for and documented in each patient and a simple scoring system based on an individualized point-based quantitative model that assigns one point per factor was used. ${ }^{14}$ Priority factors included high-risk diagnoses (age-related macular degeneration, neovascular glaucoma, recently diagnosed ischemic central retinal vein occlusion or proliferative diabetic retinopathy), monocular patients, patients who received $\geq 4$ injections in the last 6 months before the lockdown, central macular thickness (CMT) of $\geq 450$ micrometer on the last OCT image and a worsening of $\geq 2$ Snellen visual acuity lines between the last two visits before the lockdown. Accordingly, the risk scores ranged from 0 to 6 and patients were contacted after prioritization of the calls based on the scores the patients received starting with patients with the highest scores and ending with those with the lowest scores. Patients who had missing or incomplete records were excluded from data analysis.

\section{Screening for COVID-19 Exposure}

Patients were asked over the phone if they had recently experienced any of the following symptoms: high fever, difficulty breathing, loss of smell, dry cough and flu-like illness. Quarantined patients and patients with positive COVID-19 tests were postponed until they have recovered and tested negative or until their quarantine was over. Patients with active symptoms or patients who reported exposure to COVID-19 positive or to symptomatic patients in the last 14 days were advised to quarantine themselves in their homes and their information was passed on to the infection control committee which provided them with the necessary services such as testing and management, if needed, at designated COVID-19 facilities. Patients who were not reached by phone or those who declined to come in for therapy, were skipped and excluded from data analysis. Over the phone, clear instructions on how to minimize their risk for COVID-19 exposure were given and preliminary verbal consent for the visit and the IVI was obtained over the phone.

\section{Safety Precautions in the Injection Room}

A well-ventilated section from the ophthalmology clinic floor at the hospital was isolated and assigned for IVI during the lockdown. The section encompassed a large waiting room, a clinic containing a slit-lamp biomicroscope with a fixed plastic breath shield along with a phoropter and Snellen visual acuity projector, a room with only an OCT device (TOPCON 3D OCT-2000, TOPCON medical systems, Inc., Oakland, NJ) and an injection room with a single medical bed. Floors and walls in the assigned rooms were scrubbed and surfaces of tables, chairs and devices sprayed and wiped using hydrogen peroxide and ethanol-based detergents the nights before the injection days. Social distancing of 2 meters or more and the frequent use of hand disinfectants, which were available in all rooms, were strongly recommended. All patients who presented to the clinic would be checked for face masks before entering the clinic and new surgical face masks, disposable gowns and gloves were provided. All involved medical staff were selected to be experienced in IVI procedures and to have recently received in-house training in the COVID-19 infection control protocols and were all screened for exposure and symptoms of COVID-19 beforehand. The staff consisted of one consultant ophthalmologist, one ophthalmology nurse and one optician/medical photographer. All staff members were instructed to wear personal protective equipment (PPE), including N95 face masks and disposable gloves, goggles and gowns, while on duty. Chairs in the waiting room were separated by a distance of 2 meters.

One patient at a time would be assisted at multiple stations where the clinical evaluation and eventually the injection would be completed. First, subjective refraction would be performed with the phoropter and best corrected visual acuity (BCVA) documented within 10 minutes or less, then a single dose unit of Minims ${ }^{\circledR}$ tropicamide $1 \%$ eye drops was used to dilate the patient's pupil and directly discarded. A standard macular cube OCT image would then be taken in the adjoining room. Ocular examination would take place in the slit-lamp room with intraocular pressure (IOP) measured by Goldmann tonometry and the retina examined with a non-contact hand-held lens. Two tonometer's tips were available for use and were immersed in $70 \%$ alcohol after use. Injections were administered in the injection room using published expert committee guidelines including the use of topical $5 \%$ povidone iodine at the injection site and the avoidance of eyelid contact with the needle. ${ }^{15}$

\section{Statistics}

Statistical evaluations were performed using Med-Calc (version 12.3.0.0; MedCalc software, Mariakerte, Belgium) and Microsoft Excel 2013 (Microsoft, Redmond, WA). Two-tailed Student $t$-test was used to 
compare continuous variables and two-tailed Fisher's exact test was used for categorical variables. A $p$ value less than 0.05 was considered statistically significant.

\section{Results}

Between patients who were scheduled to receive IVI after the day of the lockdown (March 16th, 2020) and those who missed their IVI therapy before the lockdown, a total of 282 patients were contacted. After exclusion of patients who were not reached by phone (43), had missing or incomplete medical records (8), were symptomatic or exposed to COVID-19 patients (6), who declined to leave their homes during the active lockdown (15), were systemically unstable (10), who eventually were a noshow at their given appointments (68), a total of 132 patients received their intravitreal injections and were eventually included in the data analysis. All the injections and evaluations took place between April 12th and May 9th, 2020, after the final approval of the national infection control committee was received on April 8th, 2020.

The mean age of the patients was $59.9 \pm 13.7$ (24-84) years and 71 patients $(53.7 \%)$ were male. Compared to a daily range of 16 to $20(18.4 \pm 1.6)$ patients in the month before the lockdown, the daily number of patients receiving IVI therapy during the lockdown ranged from 2 to 10 $(6.6 \pm 2.1)$. All patients were on a pro-re-nata (PRN) regimen starting after 3 monthly loading doses. Figure 1 shows indications for IVI in our patients.

All patients and medical staff adhered to the instructions related to the infection control protocol at the designated clinics, with no related incidents or complications recorded. All patients successfully received their assigned intravitreal injection. Eighty-five patients (64.4\%) were given aflibercept $2 \mathrm{mg}$ in $0.05 \mathrm{~mL}$ and the remaining 47 patients $(35.6 \%)$ received ranibizumab $0.5 \mathrm{mg}$ in $0.05 \mathrm{~mL}$. No serious ocular or systemic adverse events related to IVI were observed at the time of injection or documented thereafter through follow up phone calls. No new exposures or COVID-19 positive cases were traced to our location or time of therapy.

Overall, patients were delayed for a mean of $6.2 \pm 1.4$ weeks from their original IVI appointment due to the lockdown (range 4-8 weeks) with 28 patients $(21.2 \%$ ) delayed for 8 weeks. The mean number of intravitreal injections received by our patients in the past 6 months prior to the lockdown was $2.2 \pm 1.5$ injections (range 1-6) with 16 patients $(12.1 \%)$ receiving $4-6$ injections and 4

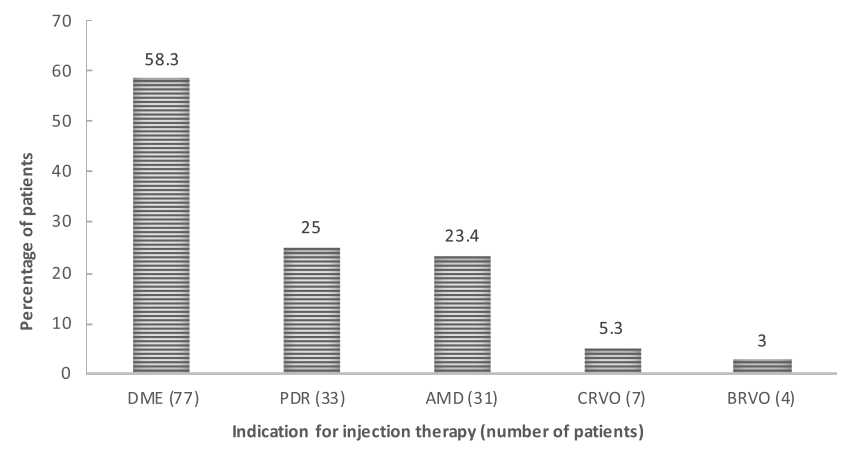

Figure I Distribution of the indications (in percentage) for intravitreal injection therapy in patients who received their injections during the COVID-19 lockdown. Abbreviations: AMD, wet age-related macular degeneration; BRVO and CRVO, branch and central retinal vein occlusion; DME, diabetic macular edema; PDR, proliferative diabetic retinopathy.

patients $(3 \%)$ receiving 6 monthly injections. Among the different indications, the mean delay and mean number of injections received in the past 6 months prior to the lockdown were $6.4 \pm 1.3$ weeks and $2.4 \pm 1.6$ injections, respectively, for DME patients, $6.6 \pm 1.0$ weeks and 2.2 \pm 1.3 injections, for PDR patients, $5.9 \pm 1.4$ weeks and 2.5 \pm 1.1 injections, for AMD patients, and $5.1 \pm 1.6$ weeks and $2.2 \pm 0.8$ injections, for RVO patients.

Since their last visits, none of the patients underwent cataract surgery and the number of pseudophakic patients was $105(79.5 \%)$. Out of the remaining 27 patients with cataract, severity grading changed in $3(11.1 \%)$ as follows: the grade changed from +1 to +2 in 2 patients and from +3 to +2 in 1 patient.

Evaluation of key clinical parameters prior and during the lockdown is detailed in Table 1 and progression in disease stage over the delay is illustrated in Table 2.

The overall number of patients who had BCVA $\leq 20$ / 200 at the last visit before the lockdown was $17(12.8 \%)$ compared to $28(21.2 \%)$ patients at the day of injection ( $p=0.101)$. Similarly, the number of patients who had a CMT $\geq 500 \mu$ on OCT before the lockdown was 16 $(11.3 \%)$ patients compared to $29(21.9 \%)$ patients on the day of the injection ( $p=0.0487)$.

When asked the first question "How would you describe your visual function at this time compared to your last visit before the lockdown?", 69 patients $(52.2 \%)$ answered "no notable change", 48 patients (36.3\%) answered "worse", 4 patients (3\%) answered "better" and $11(8.3 \%)$ patients did not participate. For patients who answered "worse", their answers to the second question: "How did the delay in treatment due to the lockdown affect your quality of life?" were as 
Table I Comparison of Visual Acuity, Macular Thickness, and Intraocular Pressure Values Before and During the COVID-19 Lockdown in Patients with Different Conditions Who Received Intravitreal Injection Therapy

\begin{tabular}{|c|c|c|c|}
\hline $\begin{array}{l}\text { Clinical } \\
\text { Parameter }\end{array}$ & $\begin{array}{l}\text { Prior to } \\
\text { Lockdown }\end{array}$ & $\begin{array}{l}\text { During } \\
\text { Lockdown }\end{array}$ & $p$ value $^{\alpha}$ \\
\hline $\begin{array}{l}\text { All patients } \\
\text { - BCVA } \\
\text { - OCT CMT } \\
\text { - IOP }\end{array}$ & $\begin{array}{l}0.44 \pm 0.31 \\
329 \pm 128 \\
13.8 \pm 2.3\end{array}$ & $\begin{array}{l}0.53 \pm 0.34 \\
370 \pm 149 \\
14.1 \pm 1.6\end{array}$ & $\begin{array}{l}0.0255 \\
0.0172 \\
0.2197\end{array}$ \\
\hline $\begin{array}{l}\text { DME } \\
\text { - } \text { BCVA } \\
\text { - OCT CMT } \\
\text { - IOP }\end{array}$ & $\begin{array}{l}0.46 \pm 0.27 \\
344 \pm 133 \\
13.6 \pm 2.2\end{array}$ & $\begin{array}{l}0.58 \pm 0.38 \\
381 \pm 153 \\
14 \pm 1.2\end{array}$ & $\begin{array}{l}0.0038 \\
0.0541 \\
0.1918\end{array}$ \\
\hline $\begin{array}{l}\text { PDR } \\
\text { - } \text { BCVA } \\
\text { - OCT CMT } \\
\text { - IOP }\end{array}$ & $\begin{array}{l}0.33 \pm 0.17 \\
313 \pm 123 \\
14.1 \pm 2.6\end{array}$ & $\begin{array}{l}0.41 \pm 0.19 \\
364 \pm 140 \\
14 \pm 1\end{array}$ & $\begin{array}{l}0.0762 \\
0.0649 \\
0.7466\end{array}$ \\
\hline $\begin{array}{l}\text { AMD } \\
\text { - } \text { BCVA } \\
\text { - OCT CMT } \\
\text { - IOP }\end{array}$ & $\begin{array}{l}0.48 \pm 0.31 \\
313 \pm 123 \\
14 \pm 2.6\end{array}$ & $\begin{array}{l}0.66 \pm 0.33 \\
371 \pm 138 \\
14 \pm 1.7\end{array}$ & $\begin{array}{l}0.0307 \\
0.0287 \\
0.9598\end{array}$ \\
\hline $\begin{array}{l}\text { RVO } \\
\text { - } \text { BCVA } \\
\text { - OCT CMT } \\
\text { - } \text { IOP }\end{array}$ & $\begin{array}{l}0.44 \pm 0.31 \\
289 \pm 125 \\
13.3 \pm 1.4\end{array}$ & $\begin{array}{l}0.6 \pm 0.4 \mid \\
426 \pm 175 \\
14.7 \pm 2.9\end{array}$ & $\begin{array}{l}0.3142 \\
0.0474 \\
0.1741\end{array}$ \\
\hline
\end{tabular}

Note: ${ }^{\alpha} p$ value calculated using two-tailed paired Student's $t$-test for comparison of the parameter's values before and after the lockdown.

Abbreviations: AMD, age-related macular degeneration; BCVA, best-corrected visual acuity expressed in logMAR value; CMT, central macular thickness expressed in micrometer; DME, diabetic macular edema; IOP, intraocular pressure expressed in $\mathrm{mmHg}$; OCT, optical coherence tomography; PDR, proliferative diabetic retinopathy; RVO, retinal vein occlusion.

Table 2 Progression in Disease Stage in Patients Who Received Intravitreal Injection Therapy During the COVID-19 Lockdown

\begin{tabular}{|l|l|l|}
\hline Clinical Stage $^{\alpha}$ & $\begin{array}{l}\text { Prior to } \\
\text { Lockdown }\end{array}$ & $\begin{array}{l}\text { During } \\
\text { Lockdown }\end{array}$ \\
\hline - Moderate NPDR & 48 & 46 \\
- Severe NPDR & 9 & 10 \\
- PDR with HRCs & 13 & 15 \\
- Inactive PDR & 20 & 19 \\
- AMD with IRF & 29 & 28 \\
- AMD with IRF and & 2 & 3 \\
- SRH & & \\
- CRVO with IRF & 5 & 4 \\
- CRVO with NVG & 2 & 3 \\
\hline
\end{tabular}

Note: ${ }^{\alpha}$ Expressed in number of patients.

Abbreviations: AMD with IRF and SRH, wet age-related macular degeneration with intra-retinal fluid and sub-retinal hemorrhage; CRVO with NVG, central retinal vein occlusion with neovascular glaucoma; NPDR, non-proliferative diabetic retinopathy; PDR with HRCs, proliferative diabetic retinopathy with high-risk characteristics. follows: $44(91.6 \%)$ patients answered "worse" again, 3 patients $(6.3 \%)$ answered "no notable change" and one patient $(2 \%)$ gave no answer.

In the last visit before the lockdown, the group of patients who answered "worse" to the first question $(n=48)$ had a mean BCVA of $0.36 \pm 0.26 \log$ MAR units and a mean CMT of $332 \pm 120 \mu$ compared to a mean BCVA of $0.48 \pm 0.25 \log$ MAR units and a mean CMT of $341 \pm 127 \mu$ in the group of patients who answered "no notable change" $(\mathrm{n}=69)$ to the same question $(p=0.03$ for BCVA comparison, $p=0.7005$ for CMT comparison).

In patients who answered "worse" to the first question, mean BCVA worsened from $0.36 \pm 0.26$ before the lockdown to $0.48 \pm 0.31 \log$ MAR units at the injection day $(p=0.0427)$ and mean CMT increased from $332 \pm 120 \mu$ before the lockdown to $391 \pm 146 \mu$ at the injection day $(p=0.0331)$.

For patients who answered "no notable change" to the first question ( $n=69)$, mean BCVA worsened from $0.48 \pm$ 0.25 before the lockdown to $0.55 \pm 0.28 \log$ MAR units at the injection day $(p=0.1237)$ and mean CMT increased from $341 \pm 127 \mu$ before the lockdown to $401 \pm 167 \mu$ at the injection day $(p=0.0189)$.

\section{Discussion}

We describe our clinical experience with administering intravitreal injections during the COVID-19 lockdown under strict adherence to infection transmission protocols. These precautions, which are consistent with recent internationally recommended retina practice guidelines and similar clinical experiences with IVI therapy from specialized retina clinics, ${ }^{7-9}$ were successfully followed by our medical staff and patients with no reported incidents. Under such circumstances, it is important to maintain frequent verbal and written communication with the patient and clear instructions regarding logistic and infection control arrangements.

It is unlikely that infection precautions and social distancing policies will cease to be implemented around the world any time soon. In fact, simulations predict that such necessary measures in healthcare, economy and social life will remains for months and years to come. ${ }^{16}$ It is therefore more reasonable for policy makers and healthcare providers to embrace new practice patterns under optimized safety measures to assure the continuation of regular medical service rather than to suspend it. We hope our positive experience would encourage other medical teams to take the lead, poised with knowledge and fortitude, in 
resuming medical service for their patients in these difficult times.

In most condition, delays and cutbacks in IVI therapy have been associated with reduced efficacy and worse visual outcomes, albeit to a variable degree. ${ }^{17-19}$ With the utilization of updated infection transmission measures, both within healthcare facilities and in the general population, the risk of spreading infection is effectively reduced to a minimum and many medical services, including ophthalmic procedures, may be safely offered to patients. $^{20-22}$

Although the primary purpose from our report was to share our successful initiative in administering IVI therapy during periods of social and logistic restrictions, we also aimed to outline the clinical features of the patients before and after the lockdown in an attempt to detect any progression that may have occurred due to the delay in therapy or evaluation. Most of our patients had diabetic exudative maculopathy and retinopathy as the indication for IVI therapy, about a quarter were AMD patients and in $<10 \%$ of cases the indication was RVO-related. While in many ophthalmic practices in the western world and many other countries, AMD may represent the largest subpopulation of patients, the proportions we had for diabetic eye disease and AMD in our patients are in keeping with the prevalence of these conditions in Jordan and the Middle East.

We noted that the frequency of IVI in our patients before the pandemic, with a mean of 2 injections per 6 months, was within the expected range demonstrated in real-life surveys, ${ }^{23,24}$ including a particular subgroup of patients, comprising about $12 \%$ in our cohort, who show modest or poor response to therapy and need monthly or near monthly injections. Unfortunately, due to the lockdown, our patients' IVI therapy was deferred until further notice. With the successful completion of our initiative, the delay in the injection schedule was limited to 6 weeks, on average, with only about $20 \%$ of patients delayed for 8 weeks. Much as progression in disease activity is foreseen after unexpected setbacks in therapy, hope was high that the shortened delay in our patients would limit or prevent progression in the disease status. However, from our 48 patients who had mild-moderate NPDR before the lockdown, 2 patients had shown progression (4.2\%), one to severe NPDR and one to PDR with vitreous hemorrhage. For those who had stable PDR before the lockdown, one patient $(5 \%)$ came with a new vitreous hemorrhage. In spite of our anticipation to report more dramatic progression in AMD or ischemic CRVO cases, new subretinal hemorrhage was documented in only 1 AMD patient (3.2\%) and no other signs of progression such as massive submacular hemorrhage, retinal pigment epithelial rips, notable enlargement of the choroidal neovascular membrane or scarring were detected in AMD patients on clinical fundoscopy or OCT. This is in contrast to recent reports from Italy demonstrating a significant rise in sightthreatening complications including massive sub-macular hemorrhage in AMD due to the shrinkage in the number of clinic visits and IVI therapies as a consequence of the national COVID-19 lockdown. ${ }^{25}$ The authors further predict short and long-term rebound effects after the end of the pandemic characterized by increased volumes of IVI and office visits. ${ }^{26}$ For CRVO cases, one patient (14.2\%), who had only macular edema before the lockdown, presented with iris neovascularization, an IOP of $36 \mathrm{mmHg}$ and enlargement in the cup-to-disc ratio, consistent with neovascular glaucoma. Regarding cataract, which was present in about $20 \%$ of the patients, an increase in the severity grade was seen in only 2 patients. In addition, in one other patient, the ophthalmologist documented a less severe grade compared to the last visit. It is therefore unlikely that cataract progression was significant enough during this relatively short delay to confound visual acuity calculations.

We documented all BCVA and OCT CMT values at the day of injection and compared them to corresponding values recorded on the last visit before the lockdown. As would be expected from delays in therapy, there was a significant overall decline in mean BCVA and a significant overall increase in mean CMT on OCT. Mean Snellen visual acuity decreased from about 20/55 to about 20/70 and the percentage of patients with BCVA $\leq 20 / 200$ increased, albeit without statistical significance. Similarly, mean CMT significantly increased by about 40 $\mu$, from 330 to $370 \mu$, and the proportion of patients with CMT values $\geq 500 \mu$ significantly doubled (from about $11 \%$ to $21 \%$ ).

Finally, we asked the patients about the effect of the lockdown on their visual function. More than half of the patients did not notice any change but more than a third thought their vision became worse. What's more, the majority of patients who thought their vision became worse also thought that their quality of life correspondingly deteriorated. When we compared clinical evaluations before and after the lockdown only for patients who answered: "worse" to the first question $(\mathrm{n}=48)$, we found 
good consistency between subjective symptoms and objective measurements, with the mean BCVA significantly worsening from about $20 / 45$ to $20 / 60$. In patients who reported "no notable change", BCVA worsened to a lesser extent after the delay, from about 20/60 to 20/70, and was without statistical significance. Baseline vision (BCVA recorded at the last visit before the lockdown) is another point of comparison between these two groups. Patients who answered "worse" had significantly better baseline visual acuities to start with compared to patients who answered: "no notable change". Apparently, patients with relatively better vision were more likely to notice and report a deterioration in their acuity than those with worse vision. Conversely, no similar pattern could be seen regarding macular edema as the mean CMT increased significantly and to the same extent (around $60 \mu$ ) in both groups. This discrepancy between visual and anatomical parameters is not surprising considering that correlation between macular thickness and visual function in the literature is typically poor to moderate. ${ }^{27,28}$

At this time and in the future, global hazards may occur and restrictions in healthcare groundwork may be applied. Our current clinical experience, which represented careful action to uphold our responsibilities to treat disease, yet without compromising our pledge to cause no harm, should be encouraged. With few similar reports published to date, 9,29 and to the best of our knowledge, our administration of IVI therapy under strict transmission control protocols during the COVID-19 lockdown, would be among the first documented clinical experiences worldwide.

Limitation to our study includes its retrospective nature with its inherent bias, limited clinical evaluations derived from only two time-points, and the lack of patient encounter in follow up to detect any complications or therapy failures. Nonetheless, several points of strength can be found in our study including the fact that our clinical experience represents a medical care initiative with collaboration at the national level between ophthalmology services and the infection control teams. In addition, the number of patients we treated $(n=132)$ was relatively high, making the demonstrated feasibility and safety in our initiative more credible and the data analysis more accurate.

In conclusion, we report our clinical experience with delivery of intravitreal injection therapy to priority patients during the COVID-19 lockdown. Clinical evaluation and intravitreal therapy were feasible and successful for all patients in a background of strict compliance with infection control precautions. Delay in therapy may compromise the visual function and quality of life of our patients. Confidence in infection control protocols and resumption of essential medical services in these difficult times is encouraged.

\section{Data Sharing Statement}

The datasets generated and/or analyzed during the current study are available from the corresponding author upon reasonable request.

\section{Ethical Approval}

Institutional ethical approval to conduct this study was duly obtained and all participants provided written informed consent (approval \# 303/2020). This research complies with the tenants of the Declaration of Helsinki.

\section{Author Contributions}

All authors made substantial contributions to conception and design, acquisition of data, or analysis and interpretation of data; took part in drafting the article or revising it critically for important intellectual content; agreed to submit to the current journal; gave final approval of the version to be published; and agree to be accountable for all aspects of the work.

\section{Disclosure}

The authors report no conflicts of interest for this work.

\section{References}

1. Perez Perez GI, Talebi Bezmin AA. Ongoing challenges faced in the global control of COVID-19 pandemic [published online ahead of print, 2020 Apr 29]. Arch Med Res. 2020;S0188-4409(20)30457-4. doi:10.1016/j.arcmed.2020.04.016

2. World Health Organization (WHO). WHO timeline - COVID-19; 2020. Available from: https://www.who.int/news-room/detail/27-042020-who-timeline - covid-19. Accessed July 30, 2020.

3. Situation Report -51 WHOC disease 2019 (COVID19). WHO report to declare pandemic. Available from: https://www.who.int/docs/default source/coronaviruse/situation-reports/20200311-sitrep-51-covid19. pdf?sfvrsn=1ba62e57_10. Accessed July 30, 2020.

4. Romano MR, Montericcio A, Montalbano C, et al. Facing COVID-19 in ophthalmology department. Curr Eye Res. 2020;45(6):653-658. doi:10.1080/02713683.2020.1752737

5. Lim LW, Yip LW, Tay HW, et al. Sustainable practice of ophthalmology during COVID-19: challenges and solutions. Graefes Arch Clin Exp Ophthalmol. 2020;21:1-10. doi:10.1007/s00417-020-04682-z

6. Saleem SM, Pasquale LR, Sidoti PA, et al. Virtual ophthalmology: telemedicine in a COVID-19 era. Am J Ophthalmol. 2020:S00029394(20)30214-2. doi:10.1016/j.ajo.2020.04.029.

7. Korobelnik JF, Loewenstein A, Eldem B, et al. Guidance for anti-VEGF intravitreal injections during the COVID-19 pandemic. Graefes Arch Clin Exp Ophthalmol. 2020;258(6):1149-1156. doi:10.1007/s00417-020-04703-x 
8. Shmueli O, Chowers I, Levy J. Current safety preferences for intravitreal injection during COVID-19 pandemic. Eye (Lond). 2020;4:1-3. doi:10.1038/s41433-020-0925-x

9. Corradetti G, Corvi F, Nguyen TV, Sadda SR. Management of neovascular age-related macular degeneration during the COVID-19 pandemia [published online ahead of print, 2020 may 29]. Ophthalmol Retina. 2020;S2468-6530(20)30207-4. doi:10.1016/j.oret.2020.05.015

10. Wong TY, Bandello F. Academic ophthalmology during and after the COVID-19 pandemic. Ophthalmology. 2020;127(8):e51-e52. doi:10.1016/j.ophtha.2020.04.029

11. Kuo IC, O'Brien TP. COVID-19 and ophthalmology: an underappreciated occupational hazard. Infect Control Hosp Epidemiol. 2020;15:1-9. doi:10.1017/ice.2020.238

12. Schulze-Bonsel K, Feltgen N, Burau H, et al. Visual acuities "hand motion" and "counting fingers" can be quantified with the freiburg visual acuity test. Invest Ophthalmol Vis Sci. 2006;47(3):1236-1240. doi:10.1167/iovs.05-0981

13. American Academy of Ophthalmology Retina-Vitreous Panel. Preferred practice pattern ${ }^{\circledR}$ guidelines. Diabetic retinopathy. San Francisco, CA: (C) American Academy of Ophthalmology; 2019. Available from: www.aao.org/ppp. Accessed June 8, 2020.

14. Caprini JA. Risk assessment as a guide for the prevention of the many faces of venous thromboembolism. Am J Surg. 2010;199(1 Suppl):S3-S10. doi:10.1016/j.amjsurg.2009.10.006

15. Avery RL, Bakri SJ, Blumenkranz MS, et al. Intravitreal injection technique and monitoring: updated guidelines of an expert panel. Retina. 2014;34(Suppl 12):S1-S18. doi:10.1097/IAE.0000000000000399

16. Adam D. Special report: the simulations driving the world's response to COVID-19. Nature. 2020;580(7803):316-318. doi:10.1038/ d41586-020-01003-6

17. Wykoff CC, Clark WL, Nielsen JS, et al. Optimizing anti-VEGF treatment outcomes for patients with neovascular age-related macular degeneration. J Manag Care Spec Pharm. 2018;24(2-aSuppl):S3S15. doi:10.18553/jmcp.2018.24.2-a.s3

18. Weiss M, Sim DA, Herold T, et al. Compliance and adherence of patients with diabetic macular edema to intravitreal anti-vascular endothelial growth factor therapy in daily practice. Retina. 2018;38 (12):2293-2300. doi:10.1097/IAE.0000000000001892

19. Heier JS, Campochiaro PA, Yau L, et al. Ranibizumab for macular edema due to retinal vein occlusions: long-term follow-up in the HORIZON trial. Ophthalmology. 2012;119(4):802-809. doi:10.1016/ j.ophtha.2011.12.005
20. Cheng VC, Wong SC, Chuang VW, et al. The role of community-wide wearing of face mask for control of coronavirus disease 2019 (COVID-19) epidemic due to SARS-CoV-2. J Infect. 2020:S0163-4453(20)30235-8. doi:10.1016/j.jinf.2020.04.024.

21. Lai THT, Tang EWH, Chau SKY, et al. Stepping up infection control measures in ophthalmology during the novel coronavirus outbreak: an experience from Hong Kong. Graefes Arch Clin Exp Ophthalmol. 2020;258(5):1049-1055. doi:10.1007/s00417-020-04641-8

22. Chandra S, Flanagan D, Hingorani M, et al. COVID19 and ophthalmology: a brief summary of the literature. Eye (Lond). 2020:1-3. doi:10.1038/s41433-020-0956-3.

23. Holz FG, Figueroa MS, Bandello F, et al. Ranibizumab treatment in treatment-naive neovascular age-related macular degeneration: results from LUMINOUS, a global real-world study. Retina. 2019:10.1097/ IAE.0000000000002670. doi:10.1097/IAE.0000000000002670.

24. Mitchell P, Sheidow TG, Farah ME, et al. Effectiveness and safety of ranibizumab $0.5 \mathrm{mg}$ in treatment-naïve patients with diabetic macular edema: results from the real-world global LUMINOUS study. PLoS One. 2020;15(6):e233595. doi:10.1371/journal.pone.0233595

25. Romano F, Monteduro D, Airaldi M, et al. Increased number of submacular hemorrhages as a consequence of COVID-19 lockdown [published online ahead of print, 2020 Jun 25]. Ophthalmol Retina. 2020:S2468-6530(20)30255-4. doi:10.1016/j.oret.2020.06.027.

26. Borrelli E, Grosso D, Vella G, et al. Impact of COVID-19 on outpatient visits and intravitreal treatments in a referral retina unit: let's be ready for a plausible "rebound effect". Graefes Arch Clin Exp Ophthalmol. 2020.

27. Deák GG, Schmidt-Erfurth UM, Jampol LM. Correlation of central retinal thickness and visual acuity in diabetic macular edema. JAMA Ophthalmol. 2018;136(11):1215-1216. doi:10.1001/jamaophthalmol.2018.3848

28. Gerendas BS, Bogunovic H, Sadeghipour A, et al. Computational image analysis for prognosis determination in DME. Vision Res. 2017;139:204-210. doi:10.1016/j.visres.2017.03.008

29. Agarwal D, Kumar A, Chhablani J, Kumar A, Sharma N. Managing intravitreal injections in adults in COVID-19 and post-COVID-19 era- initial experiences. Indian J Ophthalmol. 2020;68(6):1216-1218. doi:10.4103/ijo.IJO_1391_20
Clinical Ophthalmology

\section{Publish your work in this journal}

Clinical Ophthalmology is an international, peer-reviewed journal covering all subspecialties within ophthalmology. Key topics include: Optometry; Visual science; Pharmacology and drug therapy in eye diseases; Basic Sciences; Primary and Secondary eye care; Patient Safety and Quality of Care Improvements. This journal is indexed on PubMed

\section{Dovepress}

Central and CAS, and is the official journal of The Society of Clinical Ophthalmology (SCO). The manuscript management system is completely online and includes a very quick and fair peer-review system, which is all easy to use. Visit http://www.dovepress.com/ testimonials.php to read real quotes from published authors. 